\title{
Role of a Probiotic Strain in the Modulation of Gut Microbiota and Cytokines in Inflammatory Bowel Disease
}

\author{
V Deepak Bamola ${ }^{1}$, Divya Dubey ${ }^{1}$, Projoyita Samanta ${ }^{1}$, Saurabh Kedia $^{2}$, Vineet Ahuja ${ }^{2}$, \\ Ratna Sudha Madempudi ${ }^{3}$, Jayanthi Neelamraju ${ }^{3}$, Rama Chaudhry ${ }^{1 *}$ \\ *Corresponding author: Prof. Rama Chaudhry \\ ${ }^{1}$ Department of Microbiology, ${ }^{2}$ Department of Gastroenterology, All India Institute of \\ Medical Sciences, New Delhi, 110029 India, ${ }^{3}$ Centre for Research \& Development, Unique \\ Biotech Ltd., Plot No. 2, Phase-II, Alexandria Knowledge Park, Hyderabad, Telangana, \\ 500078, India.
}

\begin{abstract}
Human gut is one of the major niches for anaerobes which play diverse functional role in host physiology and development. Gut microbiota maintains a homeostasis and dysbiosis may contribute in pathogenesis of different diseases including inflammatory bowel disease (IBD). Probiotic intervention is an established approach to maintain microbial homeostasis for the prevention and treatment of different diseases. The efficacy of probiotic strain Bacillus clausii UBBC-07 has been demonstrated and established in various diseases but its efficacy in IBD is under reported. To assess the effect of Bacillus clausii UBBC-07 in IBD patients, a randomized controlled study was conducted. Patients were randomly allocated to either placebo or probiotic B clausii UBBC-07 for four weeks along with the standard medical treatment (SMT). Enrolled patients were evaluated before and after intervention for GI survival of the given probiotic, change in GI microbiota, change in serum cytokines, serotonin and dopamine, symptoms of disease, physical, behavioral and psychological parameters.

$B$ clausii UBBC-07 showed good survival in IBD patients in the treatment group ( $\mathrm{p}$ $<0.01)$ without any reported adverse event. Metagenomic analysis showed that the given probiotic strain was able to modulate the gut microbiota in treated group. Phylum Firmicutes was increased and phylum Bacteriodetes was decreased in the probiotic treated group. A significant increase was observed in the abundance of bacterial genera Lactobacillus, Bifidobacterium and Faecalibacterium in the probiotic treated group $(\mathrm{p}<0.01)$ as compared to placebo group. These results indicated the given probiotic strain was able to modulate that gut microbiota in probiotic treated group. Significant increase was observed in IL-10 ( $p<0.05)$ and variable decrease in the secretion of IL-1 $\beta$, TNF- $\alpha$, IL-6, IL -17 and IL -23 in probiotic treated group. Change in the concentration of serum dopamine and serotonin in the treatment group was not significant as compared to placebo group. In the treatment group a significant decrease in the symptoms of IBD and improvement in the psychological parameter to various degrees was noted. These results indicated that probiotic strain B clausii UBBC-07 affected the gut microbiota and cytokine secretion and shown efficacy in IBD patients.
\end{abstract}

Keywords: Probiotic, Bacillus clausii UBBC-07, Gut microbiome, IBD, Cytokines. 


\section{Introduction}

Human gut is one of the most complex organization of microbes and human cells. The gut microbiome is predominantly comprised of bacteria which plays vital roles in human physiology and development. Human gastrointestinal tract (GIT) is an affluent and favorable niche for anaerobes therefore the major portion of gut microbiota are composed of strict anaerobes, which outnumber the facultative anaerobes and aerobes (Bull and Plummer, 2014; Claesson et al., 2009; Finegold, 1995; Loesche, 1969; Lozupone et al., 2012). Anaerobic bacteria are very sensitivity to oxygen often unable to survive in even in very low oxygen (Albenberg et al., 2014; Rolfe et al., 1978). Microbial interventions using probiotics to maintain the microbial homeostasis is an important preventive and therapeutic approach for different diseases including inflammatory bowel disease (IBD). Each probiotic has specific effects and few probiotics viz Lactobacilli, Bifidobacteria and Bacillus strains have already been proven their beneficial effects (Andrade et al., 2020). B clausii is spore forming and resistant to low $\mathrm{pH}$ and able to survive in the intestinal environment. The spores of $B$ clausii and survive and transit through the human GI tract. Being a spore forming is the added advantage of Bacillus clausii as the probiotic of choice in the treatment of GI disorders.

Bacillus species have been used to prevent or treat various GI disorders (Zhang et al., 2015). Bacillus probiotic Enterogermina, which includes Bacillus clausii has been reported to exert beneficial effects in the treatment of gastro-intestinal diseases (Urdaci et al., 2004). Inflammatory Bowel Disease (IBD) is a disorder of the GIT and Crohn's Disease (CD) and Ulcerative Colitis (UC) are its two main types (Sarlos et al., 2014). Etiology of IBD hypothesized that the commensal flora triggers an immune response that is further modified by specific environmental factors (Acharyya, 2018; Kedia and Ahuja, 2018, 2017). Probiotics is among the therapeutic approaches which has been tested to prevent and treat gastrointestinal inflammatory disease and have been found effective to cure this lifestyle disorders and improvement in the gut health. Bacillus clausii UBBC-07 (MTCC 5472) is a commercially available safe probiotic (Lakshmi et al., 2017; Upadrasta et al., 2016). The administration of B. clausii UBBC-07 was reported safe and effective to improve the symptoms of acute diarrhea in children and adults (Neelamraju and ratna sudha, 2015; Sudha et al., 2019, 2013). In this study, we assessed the effect of the probiotic strain Bacillus clausii UBBC-07 on adults IBD patients and the study was registered with Clinical Trials Registry (CTRI) -India (RefCTRI/2019/11/022087). Survival of the given probiotic Bacillus clausii UBBC-07 in the gut, change in gut bacteria, serum cytokines, IBD symptoms, and disease severity were evaluated before and after intervention. 


\section{Results}

\section{Survival of Bacillus clausii UBBC-07:}

In both the study groups samples of the patients were evaluated for the presence of $B$ clausii UBBC-07 before and after treatment. No B clausii UBBC-07 was detected in both the groups in the pre-treatment sample. In the probiotic treated group, in the $\mathrm{UC}$ and $\mathrm{CD}$ patient $B$ clausii UBBC-07 was detected in $74.5 \%$ and $79.6 \%$ subjects respectively and the detection of given probiotic in UC and CD patients of treatment group was significant $(\mathrm{p}<0.001)$ as compared to total absence or nil detection of $B$ clausii in placebo group. These results indicated the survival of orally given probiotic strain B clausii UBBC-07 in GI tract of these subjects.

\section{Metagenomic analysis:}

Before and after intervention, Operational Taxonomic Units (OTUs) were estimated for various bacterial taxa, including phylum, class, orders, families, and genera in the UC patients of both the probiotic and placebo groups. Firmicutes, Bacteriodetes, Proteobacteria, Actinobacteria, Cyanobacteria, and Euryarchaeota were abundant in both study groups. In the treatment group, the average \% OTUs of the phylum Firmicutes were $37.58 \%$ and $48.0 \%$ in the before and after intervention samples, respectively and in the treatment group's postintervention sample, the abundance of the phylum Firmicutes increased significantly. In the treatment group, the average \% OTUs of the phylum Bacteriodetes were $35.13 \%$ and $33.14 \%$ in before and after intervention samples, respectively. In post-intervention samples, the treatment group showed a drop in OTUs of the phylum Bacteriodetes. Results are summarized in Figure 1.

Among bacterial classes no significant change in OTUs of class Bacteroidia in before and after intervention samples was observed in treatment group. In the post-intervention sample, there was a drop in class Clostridia in the treatment group, and an increase in the placebo group. In the post-intervention samples of the treatment group, there was a drop in OTUs of the class Negativicutes and an increase in the placebo group. In the treatment group, OTUs of class Bacilli were $2.86 \%$ and $11.92 \%$ in pre and post intervention samples, respectively, while in the placebo group, the average percent OTUs of class Bacilli were 10.77 $\%$ and $3.85 \%$ in pre and post intervention samples, respectively. There was a significant rise in OTUs of the class Bacilli in the treatment group's post-intervention samples and a drop in the placebo group. Results are summarized in Figure 2. 


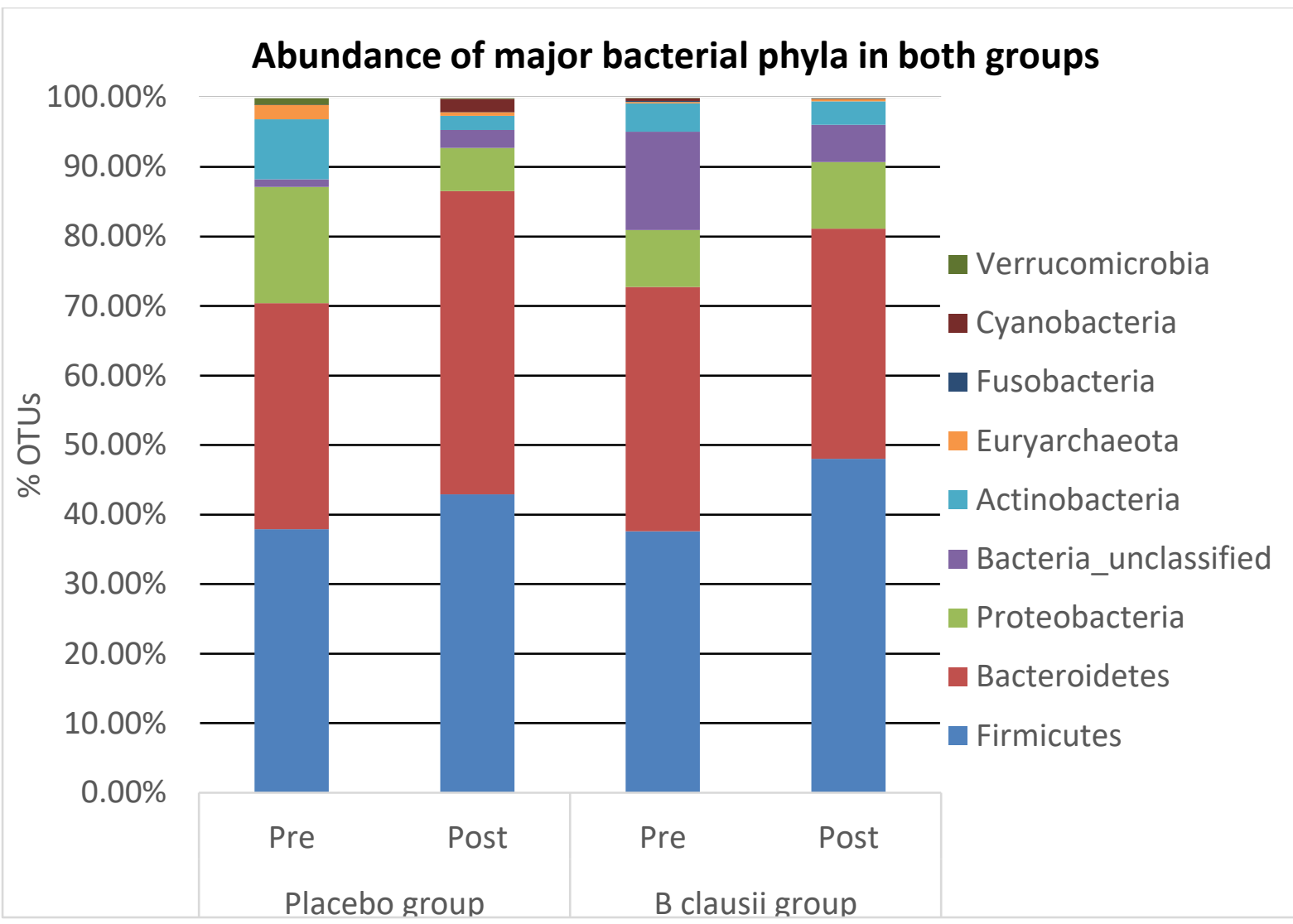

Figure 1: Abundance of major bacterial phyla (\% OTUs) in both the study group

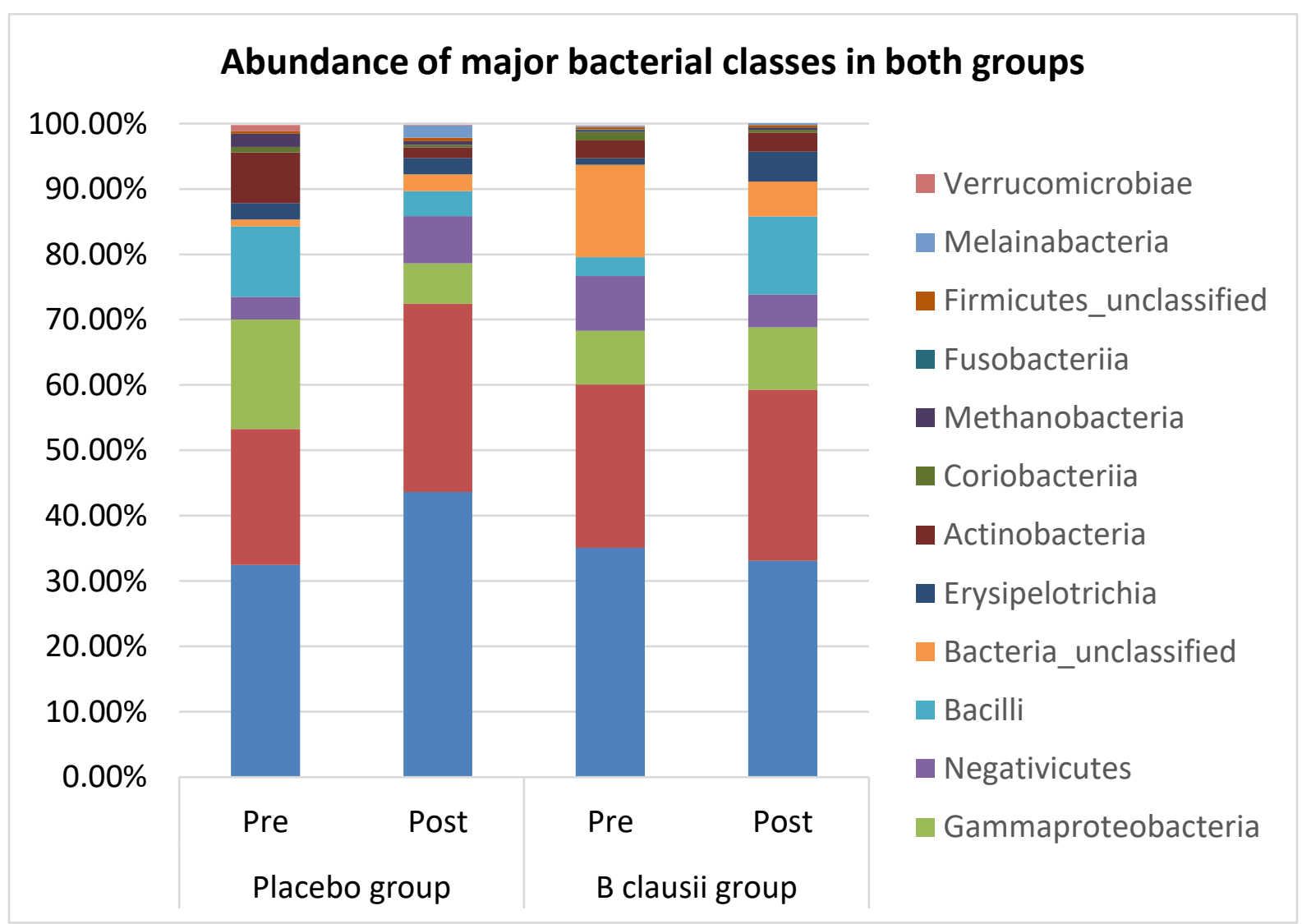

Figure 2: Abundance of major bacterial classes (\% OTUs) in both the study group 
Among bacterial orders in post-intervention samples in the treatment group, there was a drop in order Bacteroidales and an increase in the placebo group. In both groups, no significant changes in order Clostridiales were observed in post-intervention samples. After intervention there was a significant drop in order Selenomonadales in the treatment group and increase in the placebo group. In the treatment group, OTUs of order Lactobacillales was 2.79 $\%$ and $11.87 \%$ before and after intervention, respectively, and in the placebo group, 10.66 $\%$ and $3.82 \%$ before and after intervention, respectively. In post-intervention samples, there was a significant rise in the order Lactobacillales in the treatment group and a drop in the placebo group. In the treatment group, there was a significant rise in OTUs of the order Erysipelotrichales in post-intervention samples. In post-intervention samples, there was an increase in order Bifidobacteriales in the treatment group and a drop in the placebo group. Results are summarized in Figure 3.

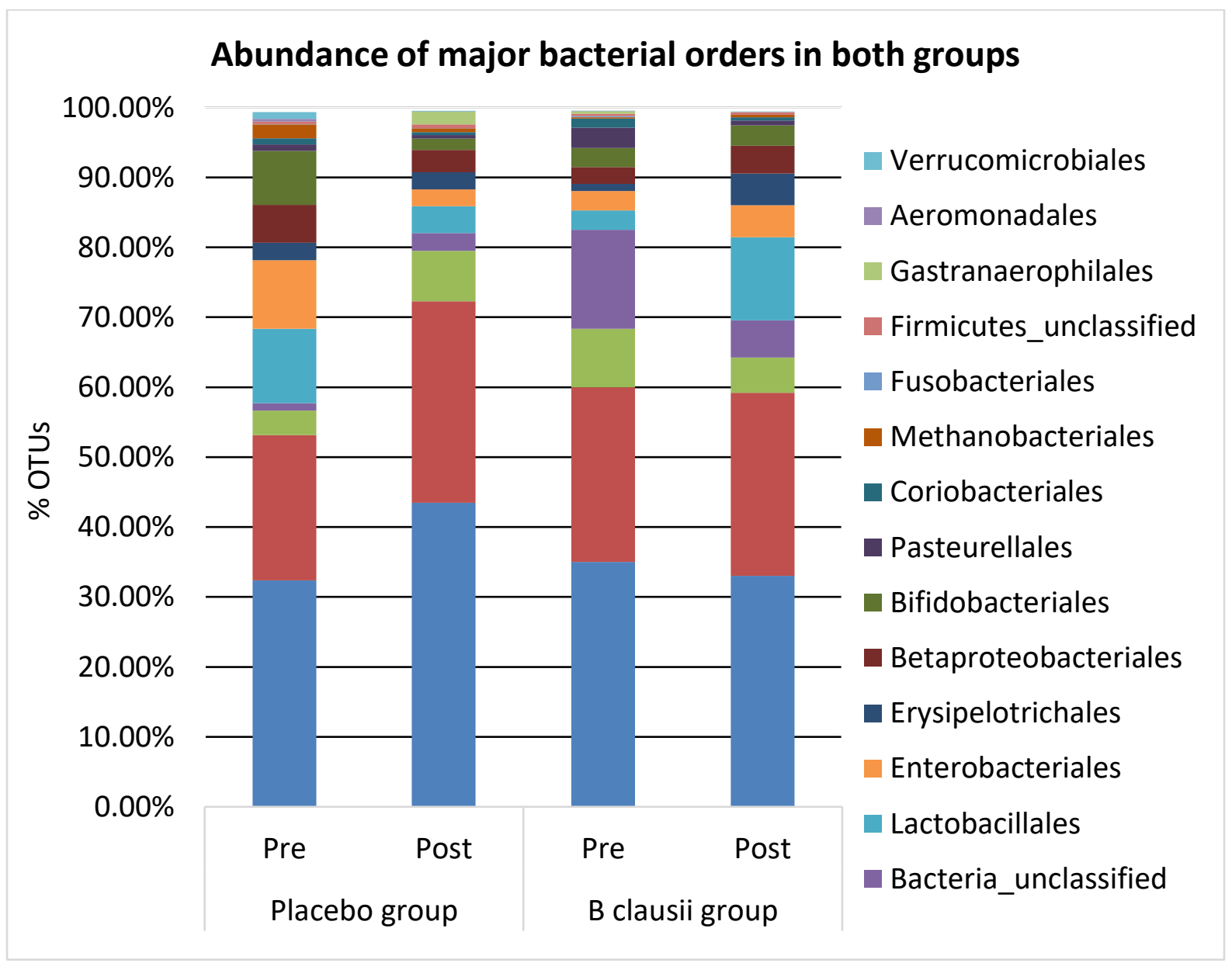

Figure 3: Abundance of major bacterial orders (\% OTUs) in both the study group 
Among bacterial families in both groups, there was an increase in OTUs of the Prevotellaceae in post-intervention samples. In post-intervention samples in the treatment group, there was an increase in the family Ruminococcaceae, while in the placebo group, there was a decrease. In post-intervention samples, there was a decrease in the family Lachnospiraceae in the treatment group and an increase in the placebo group. There was a decrease in the family Veillonellaceae in the treatment group and an increase in the placebo group, in post-intervention samples. In the treatment group, OTUs of the family Lactobacillaceae were $2.12 \%$ and $6.03 \%$, respectively, and $6.36 \%$ and $3.22 \%$ in before and after intervention, respectively. In post-intervention samples, there was a significant rise in the OTUs of Lactobacillaceae in the treatment group and a drop in the placebo group. In postintervention samples from the treatment group, there was an increase in the family Bifidobacteriaceae, while in the placebo group, there was a drop. Results are summarized in Figure 4.

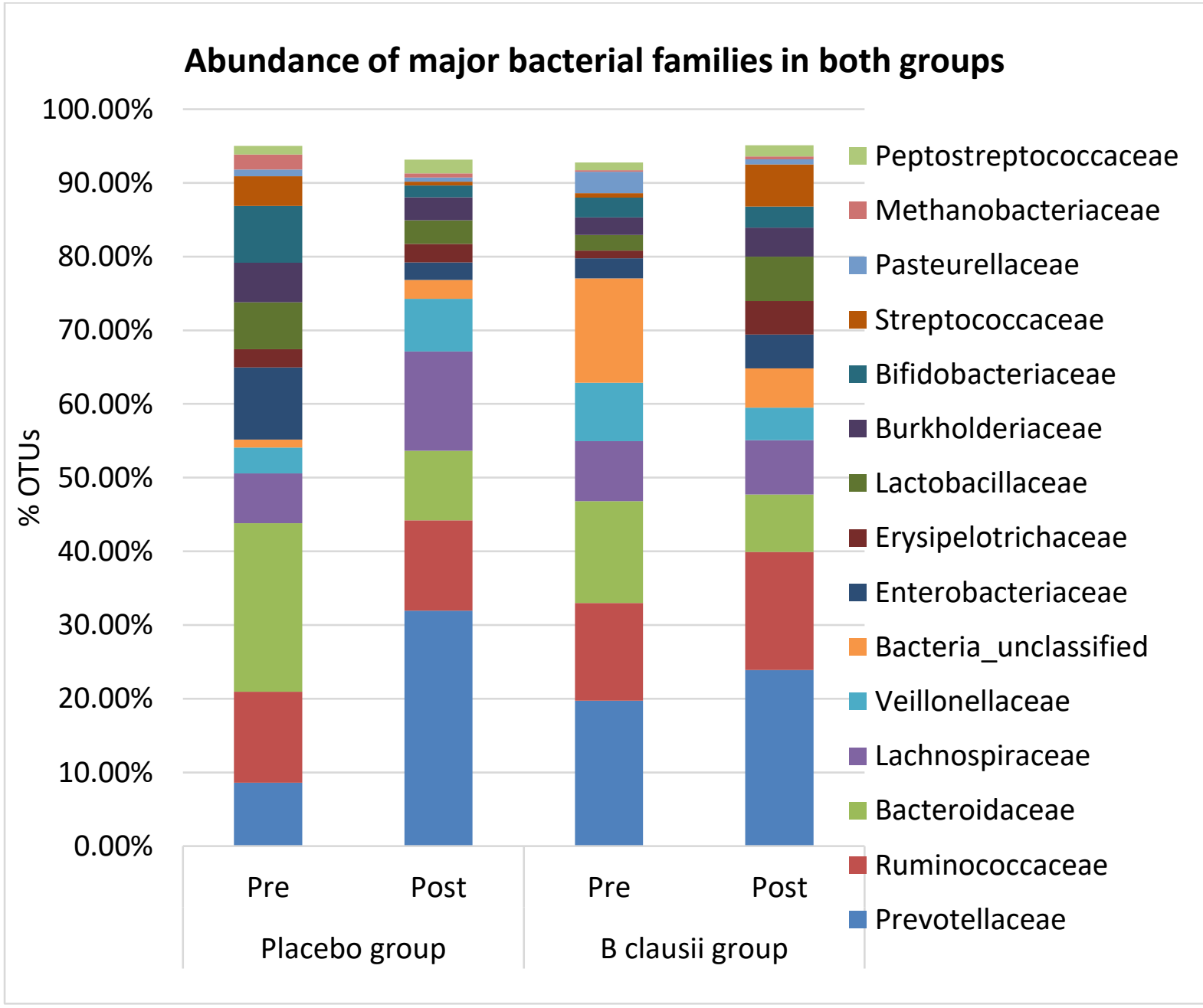

Figure 4: Abundance of major bacterial families (\% OTUs) in both the study group 
Among bacterial genera, the abundance of Lactobacillus, Bifidobacterium, Faecalibacterium, Lachnospira, Blautia, and Alistipes were increased and abundance of bacterial genera Bacteroides, Clostridia, Dialister, Megasphaera, Roseburia and Olsenella and Megamonas were decrease in the post intervention samples in the treatment group. In the post intervention samples an increase in genus Prevotella and decrease in genus Bacteroides were observed in both treatment and placebo group. Before and after intervention in the treatment group, the Faecalibacterium were $7.76 \%$ and $14.28 \%$ respectively, and in the placebo group, $9.23 \%$ and $8.01 \%$ respectively. There was a significant increase in OTUs of the genus Faecalibacterium in post intervention samples in the treatment group, while in the placebo group, there was a decrease in OTUs of the genus Faecalibacterium in post intervention samples. The average \% OTUs of the Lactobacillus were $1.69 \%$ and $5.05 \%$ in the pre and post intervention samples in the treatment group, respectively, and $7.33 \%$ and $3.49 \%$ in the placebo group, respectively. In post-intervention samples, there was a significant rise in the genus Lactobacillus in the treatment group and a drop in the placebo group. Before and after intervention the OTUs of the Bifidobacterium were $2.05 \%$ and $2.41 \%$, respectively in the treatment group and $6.03 \%$ and $1.42 \%$ before and post intervention, respectively in the treatment group. In post-intervention samples, there was an increase in the genus Bifidobacterium in the treatment group and a drop in the placebo group. Results are summarized in Figure 5.

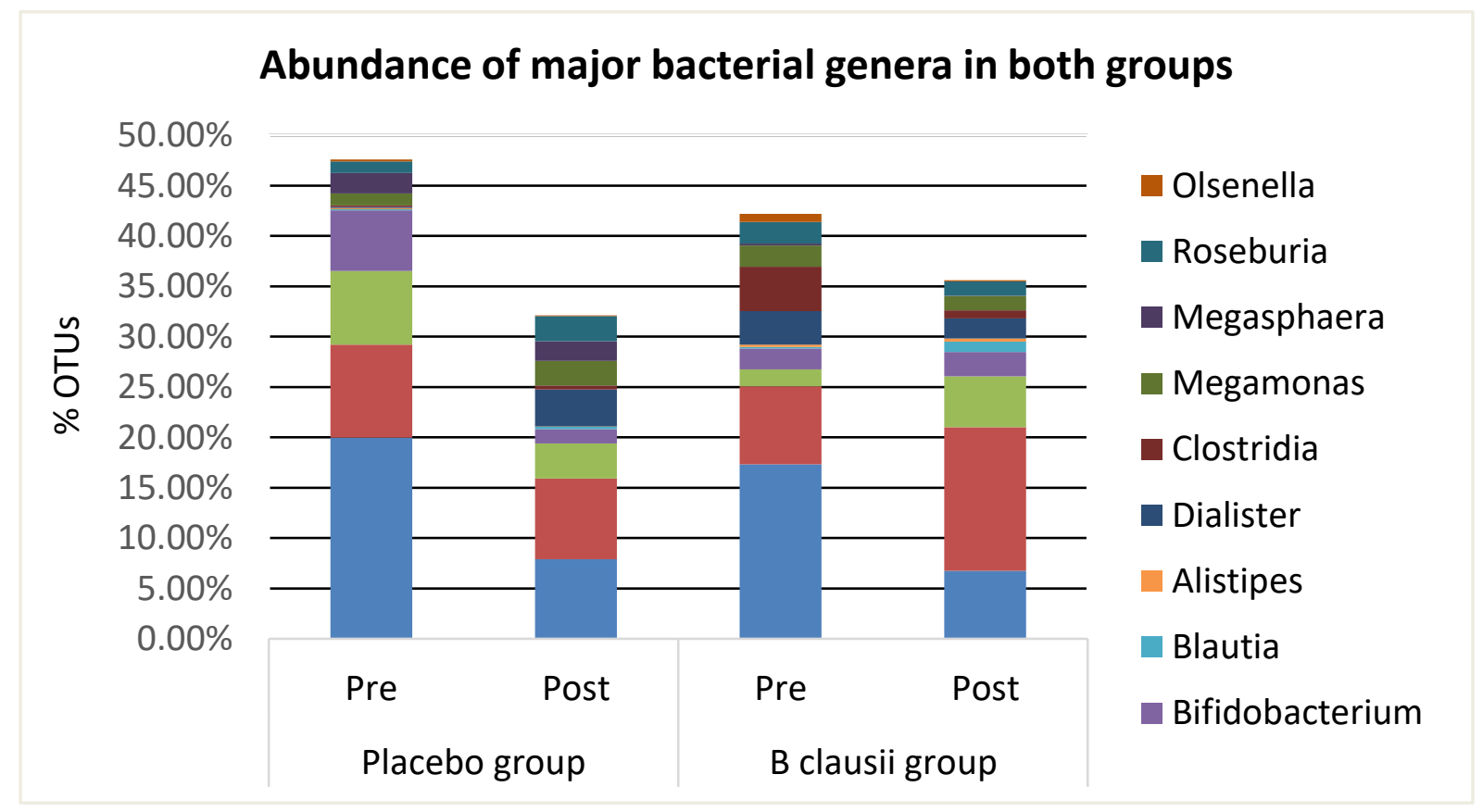

Figure 5: Abundance of major bacterial genera (\% OTUs) in both the study group 


\section{Cytokine}

Serum concentration of different cytokines were measured before and after intervention in the enrolled patients. Results indicated that the probiotic strain Bacillus clausii UBBC-07 was able to modulate the serum levels of cytokines in IBD patients. In the probiotic group serum IL10 level were $21.6 \pm 4.8 \mathrm{pg} / \mathrm{ml}$ and $31.5 \pm 5.2 \mathrm{pg} / \mathrm{ml}$ pre and post intervention in UC patients $(\mathrm{p}<0.05)$ while the difference was not significant $(\mathrm{p}=\mathrm{ns})$ in the placebo group. In the CD patient in the probiotic treated group serum IL10 level were $18.4 \pm 4.86 \mathrm{pg} / \mathrm{ml}$ and 28.4 $\pm 4.9 \mathrm{pg} / \mathrm{ml}$ pre and post intervention ( $\mathrm{p}<0.05$ ) while in the placebo group the difference was not significant $(\mathrm{p}=\mathrm{ns})$. In the treatment group serum IL6 were $44.5 \pm 5.6 \mathrm{pg} / \mathrm{ml}$ and $31.5 \pm 4.4$ $\mathrm{pg} / \mathrm{ml}$ pre and post intervention in UC patient $(\mathrm{p}<0.05)$ while in the placebo group the difference was not significant ( $\mathrm{p}=\mathrm{ns}$ ). In the UC patients, serum IL 17 level were $39 \pm 6.3 \mathrm{pg} / \mathrm{ml}$ and $24.5 \pm 4.5 \mathrm{pg} / \mathrm{ml}$ before and after intervention patient and the difference was significant ( $\mathrm{p}$ $<0.05)$ in the treatment group while in the placebo group the difference was not significant ( $\mathrm{p}$ $=\mathrm{ns})$. In the CD patient serum IL17 level were $45.4 \pm 4.8 \mathrm{pg} / \mathrm{ml}$ and $28.6 \pm 7.4 \mathrm{pg} / \mathrm{ml}$ before and after intervention and the difference was significant $(\mathrm{p}<0.05)$ in the treatment group while the difference was not significant $(\mathrm{p}=\mathrm{ns})$ in the placebo group. Serum IL23 were $898.5 \pm 54.6$ $\mathrm{ng} / \mathrm{ml}$ and $705.6 \pm 46.7 \mathrm{ng} / \mathrm{ml}$ pre and post intervention in UC patient in the treatment group (p $<0.05)$ while the difference in the placebo group was not significant (p, ns). In the CD patient serum IL23 level were $902.6 \pm 48.5 \mathrm{ng} / \mathrm{ml}$ and $734.6 \pm 40.8 \mathrm{ng} / \mathrm{ml}$ pre and post intervention in the treatment group $(\mathrm{p}<0.05)$ while in the placebo group the difference was not significant $(\mathrm{p}$, $\mathrm{ns})$. In the UC patient serum IL-1 $\beta$ level were $384 \pm 45.5 \mathrm{pg} / \mathrm{ml}$ and $246 \pm 38.4 \mathrm{pg} / \mathrm{ml}$ before and after intervention in the treatment group $(\mathrm{p}<0.05)$ while the difference was not significant $(\mathrm{p}=\mathrm{ns})$ in placebo group. In the CD patient in the treatment group serum IL-1 $\beta$ level were $375.2 \pm 36.4 \mathrm{pg} / \mathrm{ml}$ and $268 \pm 32.2 \mathrm{pg} / \mathrm{ml}$ pre and post intervention $(\mathrm{p}<0.05)$ while in the placebo group the difference was not significant $(\mathrm{p}=\mathrm{ns})$. Results are summarized in Table 1.

\section{Serum serotonin and dopamine levels:}

Before and after intervention, serum serotonin levels were $125.817 .6 \mathrm{ng} / \mathrm{ml}$ and 110.30 $12.4 \mathrm{ng} / \mathrm{ml}$, respectively, in the treatment group and $121.516 .50 \mathrm{ng} / \mathrm{ml}$ and $106.814 .65 \mathrm{ng} / \mathrm{ml}$, respectively, in the placebo group no significant difference. Before and after intervention, serum Dopamine levels were $9.253 .22 \mathrm{pg} / \mathrm{ml}$ and $10.453 .5 \mathrm{pg} / \mathrm{ml}$, respectively, in the treatment group and $10.502 .6 \mathrm{pg} / \mathrm{ml}$ and $11.43 .6 \mathrm{pg} / \mathrm{ml}$, respectively, in the placebo group with no significant difference. There was no significant difference in serum serotonin and dopamine levels in treatment and placebo groups. Results are summarized in Table 1. 
Table 1: Serum Cytokines, serotonin and dopamine levels in pre and post intervention samples in treatment and placebo group

\begin{tabular}{|c|c|c|c|c|c|c|}
\hline \multirow{2}{*}{$\begin{array}{c}\text { Cytokine } \\
(\mathrm{pg} / \mathrm{ml})\end{array}$} & \multicolumn{3}{|c|}{ Bacillus clausii UBBC-07 group } & \multicolumn{3}{|c|}{ Placebo group } \\
\hline & Pre & Post & $P$ value & Pre & Post & $P$ value \\
\hline \multicolumn{7}{|l|}{$\begin{array}{l}\mathrm{UC} \\
\text { patients }\end{array}$} \\
\hline IL-10 & $21.6 \pm 4.8$ & $31.5 \pm 5.2$ & $<0.05$ & $23.5 \pm 3.9$ & $22.6 \pm 7.5$ & NS \\
\hline IL-6 & $44.5 \pm 5.6$ & $31.5 \pm 4.4$ & $<0.05$ & $45.4 \pm 6.2$ & $40.6 \pm 4.2$ & $\mathrm{NS}$ \\
\hline IL-17 & $39 \pm 6.3$ & $24.5 \pm 4.5$ & $<0.05$ & $38.8 \pm 6.2$ & $41.9 \pm 4.6$ & NS \\
\hline IL-23 & $898.5 \pm 54.6$ & $805.6 \pm 46.7$ & NS & $906.8 \pm 68.3$ & $897.02 \pm 73.5$ & NS \\
\hline IL-1 $\beta$ & $384 \pm 45.5$ & $246 \pm 38.4$ & $<0.05$ & $352.5 \pm 67.6$ & $386.7 \pm 56.2$ & $\mathrm{NS}$ \\
\hline TNF- $\alpha$ & $65.6 \pm 6.2$ & $58.3 \pm 5.6$ & NS & $75 \pm 5.2$ & $68.5 \pm 9.5$ & NS \\
\hline \multicolumn{7}{|l|}{$\begin{array}{l}\mathrm{CD} \\
\text { patients }\end{array}$} \\
\hline IL-10 & $18.4 \pm 4.86$ & $28.4 \pm 4.9$ & $<0.05$ & $22.2 \pm 4.1$ & $25.6 \pm 6.6$ & NS \\
\hline IL-6 & $44.4 \pm 4.8$ & $38.4 \pm 7.2$ & NS & $43.5 \pm 5.6$ & $46.7 \pm 4.8$ & $\mathrm{NS}$ \\
\hline IL-17 & $45.4 \pm 4.8$ & $28.6 \pm 7.4$ & $<0.05$ & $41.4 \pm 12.4$ & $43.6 \pm 6.8$ & $\mathrm{NS}$ \\
\hline IL-23 & $902.6 \pm 48.5$ & $834.6 \pm 40.8$ & NS & $911.5 \pm 66.4$ & $875.6 \pm 56.5$ & $\mathrm{NS}$ \\
\hline IL-1 $\beta$ & $375.2 \pm 36.4$ & $268 \pm 32.2$ & $<0.05$ & $445 \pm 58.2$ & $284.5 \pm 48.5$ & $<0.05$ \\
\hline TNF- $\alpha$ & $78.4 \pm 6.2$ & $65.3 \pm 8.2$ & NS & $66.2 \pm 12.6$ & $75.5 \pm 10.8$ & NS \\
\hline \multicolumn{7}{|c|}{ Serum serotonin and dopamine in both UC and CD patients } \\
\hline $\begin{array}{l}\text { Serotonin } \\
(\mathrm{ng} / \mathrm{ml})\end{array}$ & $125.8 \pm 17.6$ & $110.30 \pm 12.4$ & NS & $121.5 \pm 16.50$ & 106.814 .65 & $\mathrm{NS}$ \\
\hline $\begin{array}{l}\text { Dopamine } \\
(\mathrm{pg} / \mathrm{ml})\end{array}$ & $9.25 \pm 3.22$ & $10.45 \pm 3.5$ & NS & $10.50 \pm 2.6$ & $11.4 \pm 3.6$ & $\mathrm{NS}$ \\
\hline
\end{tabular}

\section{Efficacy on disease symptoms}

Data was collected and recorded for various symptoms of the disease as per standard protocol. Simple Clinical Colitis Activity Index (SCCAI) score (Walmsley RS et al. 1998) was used to quantify UC disease activity. The Crohn's Disease Activity Index (CDAI) score (Best et al 1976, Sandborn et al 2002) was used to asses CD. Reduction in SCCAI score indicates the decrease in the severity of symptoms of UC and reduction in the CDAI score indicates the decrease in the severity of symptoms of CD. The SCCAI score was decreased in $42.52 \%$ and $31.58 \%$ of the UC patients post intervention in treatment and placebo group respectively and 
the difference was significant $(\mathrm{p}<0.05)$. There was no significant difference in CDAI score between treatment and placebo groups.

Table 2: Post intervention decrease in symptoms in the enrolled subjects for different physical, behavioral and psychological parameters

\begin{tabular}{|c|c|c|c|c|c|c|}
\hline \multirow{3}{*}{$\begin{array}{l}\text { Physical, } \\
\text { behavioural and } \\
\text { psychological } \\
\text { parameters }\end{array}$} & \multicolumn{6}{|c|}{ Post intervention decrease (\% of subjects) in symptoms } \\
\hline & \multicolumn{3}{|l|}{ UC patients } & \multicolumn{3}{|l|}{ CD patients } \\
\hline & $\begin{array}{l}\text { Probiotic } \\
\text { group }\end{array}$ & $\begin{array}{l}\text { Placebo } \\
\text { group }\end{array}$ & $\begin{array}{l}\mathrm{P} \\
\text { value }\end{array}$ & $\begin{array}{l}\text { Probiotic } \\
\text { group }\end{array}$ & $\begin{array}{l}\text { Placebo } \\
\text { group }\end{array}$ & $\mathrm{P}$ value \\
\hline Procrastination & $37.14 \%$ & $28.20 \%$ & $<0.05$ & $36.84 \%$ & $26.31 \%$ & $<0.05$ \\
\hline Restlessness & $28.20 \%$ & $20.51 \%$ & $<0.05$ & $21.05 \%$ & $13.33 \%$ & $<0.05$ \\
\hline Muscles stiffness & $40.0 \%$ & $35.89 \%$ & NS & $36.84 \%$ & $40.0 \%$ & NS \\
\hline Heartburn & $42.85 \%$ & $33.33 \%$ & $<0.05$ & $36.84 \%$ & $26.66 \%$ & $<0.05$ \\
\hline Headache & $37.14 \%$ & $35.89 \%$ & $\mathrm{NS}$ & $31.57 \%$ & $26.66 \%$ & $\mathrm{NS}$ \\
\hline $\begin{array}{l}\text { Shakiness or } \\
\text { tremor }\end{array}$ & $26.31 \%$ & $26.66 \%$ & NS & $42.85 \%$ & $30.76 \%$ & $<0.05$ \\
\hline Sleep problem & $42.85 \%$ & $30.76 \%$ & $<0.05$ & $36.84 \%$ & $26.66 \%$ & $<0.05$ \\
\hline $\begin{array}{l}\text { Difficulty in } \\
\text { completing work }\end{array}$ & $34.28 \%$ & $30.76 \%$ & NS & $31.57 \%$ & $33.33 \%$ & NS \\
\hline Overwhelming & $37.14 \%$ & $25.64 \%$ & $<0.05$ & $31.57 \%$ & $20.0 \%$ & $<0.05$ \\
\hline Trouble relaxing & $34.28 \%$ & $23.07 \%$ & $<0.05$ & $31.57 \%$ & $13.33 \%$ & $<0.05$ \\
\hline Nervousness & $31.42 \%$ & $30.76 \%$ & $\mathrm{NS}$ & $36.84 \%$ & $13.33 \%$ & $<0.05$ \\
\hline Depression & $25.71 \%$ & $25.64 \%$ & NS & $21.01 \%$ & $21.01 \%$ & NS \\
\hline Poor concentration & $25.64 \%$ & $28.20 \%$ & NS & $31.57 \%$ & $26.31 \%$ & NS \\
\hline Quick temper & $28.20 \%$ & $20.51 \%$ & $<0.05$ & $21.05 \%$ & $13.33 \%$ & $<0.05$ \\
\hline
\end{tabular}

\section{Physical, behavioral and psychological parameters}

Before and after intervention the enrolled IBD subjects were assessed for the different physical, behavioral and psychological symptoms. Symptoms were assessed by scores, reduction in the score indicates the decrease in the severity of symptoms and rise in the score indicates the increase in the severity of the symptom. There was no significant reduction in the complaint of muscle stiffness, heartburn, headache, shakiness or tremor, difficulty in completing work, nervousness, depression, poor concentration after intervention in UC \& CD patients in treatment group as compared to placebo group (p, ns). The complaint of sleep problems was reduced in $42.85 \%$ and $30.76 \%$ of UC patients in the treatment and placebo groups, respectively, with a significant difference between the groups $(\mathrm{p}<0.05)$. Sleep complaints were reduced in $36.84 \%$ and $26.66 \%$ of CD patients in the treatment and placebo groups, respectively, with a significant difference between the groups $(p<0.05)$. 
Procrastination was reduced in $37.14 \%$ and $28.20 \%$ of UC patients in the treatment and placebo groups, respectively, with a significant difference between the groups $(\mathrm{p}<0.05)$. Procrastination was reduced in $36.84 \%$ and $26.66 \%$ of $\mathrm{CD}$ patients in the treatment and placebo group, respectively, with a significant difference between the groups $(\mathrm{p}<0.05)$. Restlessness was reduced in $28.20 \%$ and $20.51 \%$ of UC patients in the treatment and placebo groups, respectively, with a significant difference between the groups $(\mathrm{p}<0.05)$. Restlessness was reduced in $21.05 \%$ and $13.33 \%$ of CD patients, in the treatment and placebo groups, respectively, with a significant difference between the groups $(\mathrm{p}<0.05)$. Results showed significant $(\mathrm{p}<0.05)$ decreased in the complaint of sleep problem, complaint of procrastination, complaint of overwhelming, complaint of trouble relaxing, complaint of quick temper, compliant of restlessness in the post intervention in the probiotic treated group

Safety evaluations: No adverse events were noticed, recorded, or reported during or after intervention in the trial, which further supported the safety of Bacillus clausii UBBC-07. 


\section{Discussion}

Probiotics are viable microorganisms that have a beneficial effect on health and have been used in different gastrointestinal (GI) conditions including IBD. The effects of probiotics are strains specific. Lactobacillus and Bifidbacterium are considered as good probiotic but in the harsh environment of the gut their survival is low (Keller et al., 2019). Bacillus species have potential probiotics properties (Ratna Sudha and Bhonagiri, 2012) and are considerably better than other probiotics in gastric conditions because of high heat resistance, better acid tolerance (Hyronimus et al., 2000; Lee et al., 2019). These Bacillus species may have beneficial effects in various disease including IBS, IBD, diarrhea, respiratory disorders, allergies, skin disorders, bacterial vaginosis and cancer (Pham et al 2008). The beneficial effects of these may exert through immune-modulation, competitive exclusion of pathogens and secretion of antimicrobial substances (Cunningham et al., 2021; Fuller, 1991; Indira et al., 2019; Rocchetti et al., 2021; Urdaci et al., 2004; Zommiti et al., 2020).

Bacillus clausii UBBC-07 (MTCC 5472) is well characterized probiotic strain commercially available in different formulation (Lakshmi et al., 2017). Clinical studies have shown the effectiveness of $B$. clausii UBBC-07 in the treatment of acute diarrhea in adult (Sudha et al., 2013) and children (Sudha et al., 2019). Under in vitro GIT conditions B clausii UBBC-07 were also found capable of surviving and germinating (Ahire et al., 2020). Genetic makeup of $B$. clausii UBBC07 has proven absence of toxin genes, absence of transferable antibiotic traits (Upadrasta et al., 2016). Results of the present study showed that B clausii UBBC-07 was able to survive in GI tract of IBD patients. Colonization resistance of human microbiota is an important feature where indigenous non-pathogenic bacteria suppress the growth of pathogens and provide protection to host against colonization of harmful bacteria (Kho and Lal, 2018).

Changes into human gut microbiota may trigger infections and lifestyle disorder including IBD (Gagliardi et al., 2018; Vijay and Valdes, 2021). Results of metagenomic analysis showed that phylum Firmicutes, Bacteriodetes, Proteobacteria, and Actinobacteria were more abundant in the patients of both the study group and this finding was in agreement that the Indian gut microbiome is dominated by these bacterial phyla (Gupta et al., 2019; Jain et al., 2018). After intervention, in the probiotic treated group, the abundance of Firmicutes was increased and abundance of Bacteriodetes was decreased. In post-intervention samples, the abundance of Lactobacillus, Bifidobacterium, and Faecalibacterium increased in the treatment group and decreased in the placebo group. B clausii UBBC-07 was able to increase 
beneficial Lactobacilli in treatment group which is in agreement with another reported study where Bacillus species was able to increase the abundance of Bifidobacteria and Lactobacillus (Ara et al., 2002). Studies have reported that Bacillus species is capable of restoring the microbial imbalance (Hempel et al., 2012) by promoting the growth of Lactobacillus and Bifidobacterium (Cao et al., 2020). It is postulated that Bacillus species consume free oxygen in the intestine and inhibit redox processes, providing an anaerobic and acidic environment that is unfavorable to many pathogens (Cao et al., 2020). Lactobacillus and Bifidobacteria are among the first bacteria to colonize infants and have been linked to a variety of health benefits (Feng et al., 2015; Flint et al., 2012). An increase in abundance of genus Faecalibacterium in post intervention samples in treatment group and decrease in placebo group was observed which was in agreement with previously reported decreased abundance of Faecalibacterium in IBD (Becker et al., 2015; Wang et al., 2014). Lactobacillus, Bifidobacterium, and Faecalibacterium have been reported to induced up-regulation of the anti-inflammatory cytokine, and down-regulation of inflammatory cytokines in the host to modulate mucosal inflammation (Joossens et al., 2011; Sokol et al., 2008).

Consumption of $B$ clausii UBBC-07 was reported safe and effective in acute diarrhea in adults (Sudha et al., 2013) and children (Sudha et al., 2019). This is the first study to assess the efficacy of Bacillus clausii UBBC-07 on adult IBD patients. B clausii UBBC-07 was able to reduce the severity of symptoms of disease to various degrees in IBD patients and these findings matched with those of another study, where a probiotic (VSL\#3) lowered inflammatory cytokine expression and reduce the severity of symptom in UC patients (Tursi et al., 2010). Probiotics can reduce inflammation and disease symptoms by alteration of the mucosal immune system, competitive exclusion of pathogens, production of antimicrobial factors (Schlee et al., 2008). Probiotic intervention may improve GI symptoms due to antimicrobial properties (Didari et al., 2015; Gareau et al., 2010). Probiotics normalize bowel movements and reduce visceral hypersensitivity (Gareau et al., 2010; Korterink et al., 2014). Cytokines are important signals in the gut immune system that are known to and mediate local and systemic inflammation (Sanchez-Munoz et al., 2008). The GI microbiota is thought to be the primary cause of an improper host immune response (Kho and Lal, 2018).

Changes in the serum levels of anti-inflammatory cytokine and pro-inflammatory cytokines is reported in GI disorders (Bennet et al., 2016). B clausii UBBC-07 was able to upregulate the secretion of serum IL-10. An inactivation of IL-10 leads to increased release of IL12 and IFN- $\gamma$. Low level of IL-10 has been observed in inflamed tissues and granulomas of IBD. The observed results of our study indicated that $B$ clausii UBBC-07 has significantly 
decreased the level of IL-6 in IBD patients. IL-6 is a pleiotropic pro-inflammatory cytokine which induces the terminal differentiation of $\mathrm{B}$ cells, enhances the secretion of immunoglobulin, and also elevates the secretion of acute-phase proteins. Literature reported that the expression of IL- 6 is found to be expressed in high level in the patients of IBD indicates its role in the intestinal inflammation of IBD. Clinical studies reported the expression of IL-6 as an indication in the pathogenesis of mesangial proliferative glomerulonephritis, multiple myeloma, rheumatoid Arthritis (Liu et al., 2019; Weber et al., 2016).

We have observed the reduction in the level of IL-17 and IL-23 in the treatment group. IL 17 is an important pro-inflammatory cytokine produced by a T-cell subset. IL-23 amplifies the Th17 cell responses and leads to gut inflammation. It is evident that IL-23 helps in enhancing the synthesis of both Th1 and Th17-cytokines in IBD. We have observed the decreased secretion of serum IL- $1 \beta$ in the treatment group. IL-1 $\beta$ is a pro-inflammatory cytokine and an increased production of IL-1 $\beta$ may lead to inflammation. We have observed a decrease in the secretion of serum TNF- $\alpha$ in the treatment group. TNF- $\alpha$ is a pro-inflammatory cytokine which enhanced expression of IL-2, IL-1 $\beta$ and IL-6 (Liu et al., 2019; Mavropoulou et al., 2020). It has been reported that the macrophages of IBD patients are having the high expression of TNF alpha and leads to the intestinal inflammation. Clinical studies have reported an improvement in $\mathrm{CD}$ patients treated with anti-TNF- $\alpha$ therapy such as infliximab, adalimumab and certolizumab pegol (Sanchez-Munoz et al., 2008). Observed results suggested that probiotic Bacillus clausii UBBC-07 has the potential to modulate the secretion of cytokines and gut inflammation.

\section{CONCLUSIONS}

Bacillus clausii UBBC-07 showed good survival in IBD patients without any reported adverse event. An increase in the abundance of phylum Firmicutes and decrease in the Bacteriodetes was observed after intervention in the probiotic treated group. Significant increase was observed in the bacterial genera Lactobacillus, Bifidobacterium and Faecalibacterium in post intervention sample in the probiotic treated group. A significant increase in the IL-10 and variable decreased in the secretion of IL- 6 , IL-1 $\beta$, TNF- $\alpha$, IL - 17 and IL -23 was observed in probiotic treated group. Probiotic strain B clausii UBBC-07 shown efficacy in IBD patients by modulating gut microbiota, cytokines, decreased in the symptoms of IBD and improvement in the psychological parameter to various degrees. 


\section{'CONSORT Flow Chart'}

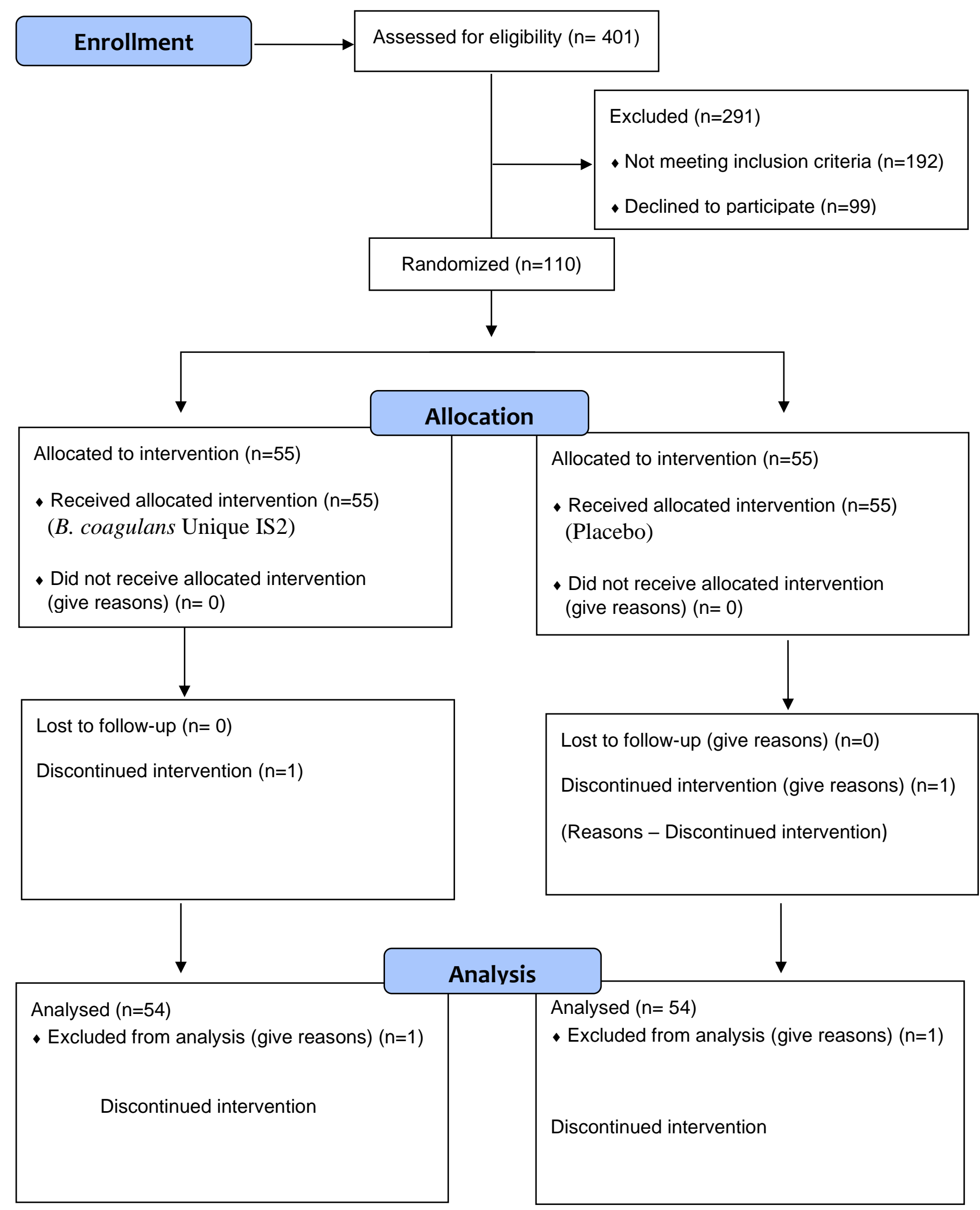




\section{Material and Methods}

- Study design: A double blind, randomized, placebo-controlled study was conducted with intervention of 4 week to assess the efficacy of Bacillus clausii UBBC-07 (MTCC 5472) in IBD patient. The randomization was generated by the nQuery clinical trial design sample size software in a 1:1 ratio. It was comprised of before intervention baseline visit (visit-1, week 0 / day 0), and post intervention visit (visit-2, 1 week after completion of intervention).

- Study site and ethical approval: The study was conducted in the tertiary care hospital after ethical approval from Institute Ethics Committee of All India Institute of Medical Sciences, New Delhi, India (Ref - IEC.478/07.10. 2016.OP-7). Before enrollment in the study, a written informed consent from each enrolled patient.

- Subject selection: Adult patients of ulcerative colitis (UC) and Crohn's disease (CD) IBD of age between 18-60 years under standard medical treatment (SMT) were enrolled in the study. To quantify UC disease activity Simple Clinical Colitis Activity Index (SCCAI) score (Walmsley et al., 1998) was used. SCCAI score is a validated symptom based clinical scoring index which is used by the clinicians to quantify UC disease activity and has a good correlation with disease activity indices. SCCAI score along with other symptoms of all the enrolled UC patients were assessed before and after intervention and UC patient with Mild to moderate severity were included in this study. The Crohn's Disease Activity Index (CDAI) is a validated scoring method to assess disease severity which has been developed and used for long (Best et al 1976). In CD severe disease is defined as a CDAI >450, moderate Crohn's is CDAI 220-450, mild Crohn's is CDAI 150-220, and clinical remission is defined as a CDAI $<150$ (Sandborn et al., 2002). In this study the CD patient with mild to moderate severity were included. 5-aminosalicylic acid (5-ASA) - Sulfasalazine (3 grams/ day) or Mesalamine $800 \mathrm{mg}$ orally 3 times a day was SMT for the enrolled patient in this study.

- Inclusion criteria: (a) Subject clinically diagnosed with Ulcerative Colitis (UC) or, Crohn's Disease (CD), (c) UC or CD patients of the age between 18-60-year of either sex (d) patient ready to give written consent to participate in the study, (e) subject receiving SMT and attending out patient department (OPD) of the hospital.

- Exclusion criteria: (a) Known case of any gastrointestinal disease other than IBD, (b) known case of any carcinoma (c) known case of immunodeficiency disorder, (d) subject is on any probiotic drug or consumed any probiotic in the last one month, (e) subject not taking food through oral route. 
- Sample size: SAS software was used to calculate the sample size. To detect the presence of a proportion difference, the assumption was made that a minimum of 116 subjects to be screened and 94 to be recruited to evaluate the primary endpoint. When the overall response is minimum $30 \%$ at a significant level of 0.05 this will provide $80 \%$ power to reject the null hypothesis.

- Randomization: Subjects were randomized into two arms (Bacillus clausii UBBC-07 - 2 billion cfu twice in a day or placebo twice in a day). Randomization was conducted using opaque sealed envelopes that were indistinguishable between groups. The codes were kept blinded and given to the enrolled subjects based on the randomization numbers.

- Enrolment of subjects: After screening as per inclusion and exclusion criteria, a total of 110 subjects were recruited. During baseline visit (day 0) complete medical history, medications, physical examination and vital signs including pulse rate, respiratory rate, blood pressure and temperature were assessed during hospital visit. FDA / DCGI / FSSAI approved probiotic strain Bacillus clausii UBBC-07 (MTCC 5472) was used as an intervention agent in this study. Enrolled subjects were given probiotic (Bacillus clausii UBBC-07, 2 billion-CFU/capsule) or placebo (identical to the probiotic capsule but contained only excipient, maltodextrin) twice in day for 4 weeks. The compliance of drug and dose were followed by observation and telephonic follow-up.

- Data analysis: In this paper we are reporting the results of the study on IBD patients. Data of 54 subjects were analyzed in each group. In probiotic treated group 19 patients of CD and 35 of UC were included. In placebo group 15 patients of CD and 39 of UC were included. The Stata statistical software (Version 14, USA) was used for statistical evaluations. Subject assessment was evaluated as frequency distribution and significance was assessed with chisquare test. A $p$ value $<0.05$ was considered as statistically significant.

- Outcome measures: The outcomes were measured by (i) detection of probiotic Bacillus clausii UBBC-07, (ii) alteration in gut microbiota, (iii) alteration in concentration of serum cytokines, (iv) alteration in serum concentration of dopamine and serotonin, (v) improvement in disease symptoms, (vi) assessment of physical and psychological parameters. For the evaluation of physical, behavioral and psychological parameters all the enrolled subjects were asked to answer the questionnaire as per the Hopkins Symptom Checklist (HSCL): A self-report symptom inventory (Derogatis et al., 1974; Kleppang and Hagquist, 2016). The parameters included were headache, heartburn, muscle stiffness, shakiness, sleep problem, procrastination, overwhelming, difficulty in completing work, nervousness, restlessness, poor concentration, feeling of depression, quick temper and 
trouble relaxing. These symptoms were assessed based on scores. The rise in the score indicating an increase in the severity of the symptom and drop in the score indicating the decrease in the severity of symptoms.

- Safety evaluation: Safety of the given probiotic was assessed by reporting of adverse event by the enrolled subject. During hospital visit, monitoring of vital signs including heart rate, respiratory rate, temperature and blood pressure were measured.

- Sample collection and processing: A stool sample and a blood sample were collected from each enrolled subject before and after intervention. A fresh stool sample was collected in a sterile container and a blood sample in a plain vial was collected from each enrolled subject. After collection, the stool samples were aliquoted and processed for microbial identification and bacterial DNA isolation. Blood samples were also processed for serum separation. Serum was separated from the whole blood samples collected in the plain vial by centrifuging at 3000-4000 rpm for 5-10 min. The serum samples were used for cytokine assays and stored at $-80^{\circ} \mathrm{C}$ till further use.

- Microbial detection: Mueller Hinton (MH) broth, MH agar (Difco Laboratory, Detroit, MI) and Chrome Bacillus agar (Hi Media) were used to detect Bacillus strain. The stool sample were incubated for 24 hours at $37^{\circ} \mathrm{C}$ in $\mathrm{MH}$ broth and then plated on $\mathrm{MH}$ agar plate and Chrome Bacillus agar to isolate the Bacillus clausii UBBC-07. To detect Lactobacillus species de Man, Rogosa and Sharpe (MRS) broth and agar (Difco Laboratory, Detroit, MI) was used. The stool sample were incubated for 48 hours at $37^{\circ} \mathrm{C}$ in MRS broth in Anaerobic Glove Box (Anaerobic Workstation-Whitley DG250-DonWhitley Scientific, United Kingdom) in anaerobic condition and then plated on MRS agar plate. Standard microbial culture and biochemical method and Matrix-assisted laser desorption /ionization- (MALDIand the mass analyzer is time-of-flight (TOF) analyzer (bioMérieux Inc, USA) were used to identify the isolated colonies were identified. The isolated organisms were also checked by molecular method.

- Molecular detection: Bacillus clausii was checked by $16 \mathrm{~S}$ rDNA sequencing using direct PCR using Thermal Cycler (Applied Biosystems, USA) with the in-house designed primers: forward 5'- CCTTGACGGTACCTCACCAC -3' and reverse 5'AAGCCCAATCTCTTGGGTGG -3' with the product size 299bp. The sequence similarity of primer was also checked using BLAST match (NCBI) which was 98\%. After standardization bacterial DNA samples were amplified using standard PCR and PCR product was checked by the electrophoresis and Gel Doc System (BioRad, USA). For positive control a known strain of Bacillus clausii UBBC-07 was used. 
- DNA isolation for metagenomic analysis: Enrolled patients were asked to collect a fresh stool sample in provided sterile container on both pre and post intervention visit. Total bacterial DNA from stool sample was extracted using QIAamp DNA Stool Mini Kit (Qiagen) with some modification (Bamola et al., 2017). Extracted DNA samples were checked and quantified by Nanodrop (TECAN Nano quant) and DNA samples of UC patients were processed for metagenomic analysis.

- Sequencing: Metagenomic analysis was performed on Illumina MiSeq ${ }^{\circledR}$ sequencing system (Illumina, San Diego, CA, USA). The analysis was carried out as per reported methodology (Ahmed et al., 2020). V3-V4, hyper variable regions of $16 \mathrm{~S}$ rRNA were amplified by V3-V4F (CCTACGGGNGGCWGCAG) and V3-V4R (GACTACHVGGGTATCTAATCC) primers. PCR amplification of DNA was carried out by KAPA HiFi HotStart Ready Mix as per standard protocol. The amplicons were purified using Ampure beads to remove unused primers and product was amplified with Illumina primers to generate sequencing libraries. Qubit dsDNA assay kit and Illumina Miseq with 2x300PE sequencing kit were used for libraries preparation and sequencing (Ahmed et al., 2020). FastQC and MultiQC were used to check sequence data quality. Only QC passed reads were used in mothur for pairing and a known reference and UCHIME algorithm was used for chimeric sequence identification. Operational Taxonomic Unit (OTUs) and abundance was calculated using Silva v.132 database. Chao1 and ACE, Shannon, Simpson, and Fisher indices were used for richness and relative abundance. To assess the difference among OTUs abundance between groups, Kruskal-Wallis rank sum test was used.

- ELISA for Cytokines, serum serotonin and dopamine: A blood sample was drawn from each enrolled subject and serum was separated. The separated serum samples were assayed for the concentration of IL10, IL6, IL 1 $\beta$, TNF, IL17, IL23, dopamine and serotonin as per manufacture's instruction (Fine Test, Fine Biotech Co. Ltd). In brief, (i) test samples and standards were added in 96 well coated plates, (ii) plates were incubated at $37^{\circ} \mathrm{C}$ for $90 \mathrm{~min}$ and wells were washed with wash buffer, (iii) secondary antibody were added, (iv) plates were incubated at $37^{\circ} \mathrm{C}$ for $60 \mathrm{~min}$ and wells were washed with wash buffer, (v) HRP was added (vi) plates were incubated at $37^{\circ} \mathrm{C}$ for $30 \mathrm{~min}$ and multiple washings were done, (vii) TMB substrates were added to visualize HRP reaction, (viii) absorbance was recorded at 450 nm using Nanodrop, Nanoquant Infinite M 200 Pro -microplate reader (Texan, Austria $\mathrm{GmbH})$. 


\section{References}

Acharyya, B., 2018. Incidence of Inflammatory Bowel Disease in a Tertiary Centre of Eastern India. Advanced Research in Gastroenterology \& Hepatology 9. https://doi.org/10.19080/ARGH.2018.09.555774

Ahire, J.J., Kashikar, M.S., Madempudi, R.S., 2020. Survival and Germination of Bacillus clausii UBBC07 Spores in in vitro Human Gastrointestinal Tract Simulation Model and Evaluation of Clausin Production. Frontiers in Microbiology 11.

Ahmed, N., Daniel, B., Varghese, J., Evangeline, R., Jose, T., 2020. Oropharyngeal microbiome of an HIV-positive patient. Microbial Pathogenesis 139, 103805. https://doi.org/10.1016/j.micpath.2019.103805

Albenberg, L., Esipova, T.V., Judge, C.P., Bittinger, K., Chen, J., Laughlin, A., Grunberg, S., Baldassano, R.N., Lewis, J.D., Li, H., Thom, S.R., Bushman, F.D., Vinogradov, S.A., $\mathrm{Wu}$, G.D., 2014. Correlation between intraluminal oxygen gradient and radial partitioning of intestinal microbiota. Gastroenterology 147, 1055-1063.e8. https://doi.org/10.1053/j.gastro.2014.07.020

Andrade, J.C., Almeida, D., Domingos, M., Seabra, C.L., Machado, D., Freitas, A.C., Gomes, A.M., 2020. Commensal Obligate Anaerobic Bacteria and Health: Production, Storage, and Delivery Strategies. Frontiers in Bioengineering and Biotechnology 8.

Ara, K., Meguro, S., Hase, T., Tokimitsu, I., Otsuji, K., Kawai, S., Ito, S., Iino, H., 2002. Effect of Spore-bearing Lactic Acid-forming Bacteria ( Bacillus coagulans SANK 70258) Administration on the Intestinal Environment, Defecation Frequency, Fecal Characteristics and Dermal Characteristics in Humans and Rats. Microbial Ecology in Health and Disease 14, 4-13. https://doi.org/10.1080/089106002760002694

Bamola, V.D., Ghosh, A., Kapardar, R.K., Lal, B., Cheema, S., Sarma, P., Chaudhry, R., 2017. Gut microbial diversity in health and disease: experience of healthy Indian subjects, and colon carcinoma and inflammatory bowel disease patients. Microb Ecol Health Dis 28, 1322447. https://doi.org/10.1080/16512235.2017.1322447

Becker, C., Neurath, M.F., Wirtz, S., 2015. The Intestinal Microbiota in Inflammatory Bowel Disease. ILAR J 56, 192-204. https://doi.org/10.1093/ilar/ilv030

Bennet, S.M.P., Polster, A., Törnblom, H., Isaksson, S., Capronnier, S., Tessier, A., Le Nevé, B., Simrén, M., Öhman, L., 2016. Global Cytokine Profiles and Association With Clinical Characteristics in Patients With Irritable Bowel Syndrome. Am J Gastroenterol 111, 1165-1176. https://doi.org/10.1038/ajg.2016.223

Bull, M.J., Plummer, N.T., 2014. Part 1: The Human Gut Microbiome in Health and Disease. Integr Med (Encinitas) 13, 17-22.

Cao, J., Yu, Z., Liu, W., Zhao, J., Zhang, H., Zhai, Q., Chen, W., 2020. Probiotic characteristics of Bacillus coagulans and associated implications for human health and diseases. Journal of Functional Foods 64, 103643. https://doi.org/10.1016/j.jff.2019.103643

Claesson, M.J., O’Sullivan, O., Wang, Q., Nikkilä, J., Marchesi, J.R., Smidt, H., de Vos, W.M., Ross, R.P., O'Toole, P.W., 2009. Comparative analysis of pyrosequencing and a phylogenetic microarray for exploring microbial community structures in the human distal intestine. PLoS One 4, e6669. https://doi.org/10.1371/journal.pone.0006669

Cunningham, M., Azcarate-Peril, M.A., Barnard, A., Benoit, V., Grimaldi, R., Guyonnet, D., Holscher, H.D., Hunter, K., Manurung, S., Obis, D., Petrova, M.I., Steinert, R.E., Swanson, K.S., van Sinderen, D., Vulevic, J., Gibson, G.R., 2021. Shaping the Future of Probiotics and Prebiotics. Trends Microbiol 29, 667-685. https://doi.org/10.1016/j.tim.2021.01.003 
Derogatis, L.R., Lipman, R.S., Rickels, K., Uhlenhuth, E.H., Covi, L., 1974. The Hopkins Symptom Checklist (HSCL). Psychological Measurements in Psychopharmacology 7, 79-110. https://doi.org/10.1159/000395070

Didari, T., Mozaffari, S., Nikfar, S., Abdollahi, M., 2015. Effectiveness of probiotics in irritable bowel syndrome: Updated systematic review with meta-analysis. World $\mathbf{J}$ Gastroenterol 21, 3072-3084. https://doi.org/10.3748/wjg.v21.i10.3072

Feng, Q., Liang, S., Jia, H., Stadlmayr, A., Tang, L., Lan, Z., Zhang, D., Xia, H., Xu, Xiaoying, Jie, Z., Su, L., Li, Xiaoping, Li, Xin, Li, J., Xiao, L., Huber-Schönauer, U., Niederseer, D., Xu, Xun, Al-Aama, J.Y., Yang, H., Wang, Jian, Kristiansen, K., Arumugam, M., Tilg, H., Datz, C., Wang, Jun, 2015. Gut microbiome development along the colorectal $\begin{array}{llll}\text { adenoma-carcinoma } & \text { sequence. Nat Commun } 6528 .\end{array}$ https://doi.org/10.1038/ncomms7528

Finegold, S.M., 1995. Anaerobic infections in humans: an overview. Anaerobe 1, 3-9. https://doi.org/10.1016/s1075-9964(95)80340-8

Flint, H.J., Scott, K.P., Duncan, S.H., Louis, P., Forano, E., 2012. Microbial degradation of complex carbohydrates in the gut. Gut Microbes 3, 289-306. https://doi.org/10.4161/gmic.19897

Fuller, R., 1991. Probiotics in human medicine. Gut 32, 439-442. https://doi.org/10.1136/gut.32.4.439

Gagliardi, A., Totino, V., Cacciotti, F., Iebba, V., Neroni, B., Bonfiglio, G., Trancassini, M., Passariello, C., Pantanella, F., Schippa, S., 2018. Rebuilding the Gut Microbiota Ecosystem. Int J Environ Res Public Health 15, E1679. https://doi.org/10.3390/ijerph15081679

Gareau, M.G., Sherman, P.M., Walker, W.A., 2010. Probiotics and the gut microbiota in intestinal health and disease. Nat Rev Gastroenterol Hepatol 7, 503-514. https://doi.org/10.1038/nrgastro.2010.117

Gupta, A., Dhakan, D.B., Maji, A., Saxena, R., P.K, V.P., Mahajan, S., Pulikkan, J., Kurian, J., Gomez, A.M., Scaria, J., Amato, K.R., Sharma, A.K., Sharma, V.K., 2019. Association of Flavonifractor plautii, a Flavonoid-Degrading Bacterium, with the Gut Microbiome of Colorectal Cancer Patients in India. mSystems. https://doi.org/10.1128/mSystems.00438-19

Hempel, S., Newberry, S.J., Maher, A.R., Wang, Z., Miles, J.N.V., Shanman, R., Johnsen, B., Shekelle, P.G., 2012. Probiotics for the prevention and treatment of antibioticassociated diarrhea: a systematic review and meta-analysis. JAMA 307, 1959-1969. https://doi.org/10.1001/jama.2012.3507

Hyronimus, B., Le Marrec, C., Sassi, A.H., Deschamps, A., 2000. Acid and bile tolerance of spore-forming lactic acid bacteria. Int J Food Microbiol 61, 193-197. https://doi.org/10.1016/s0168-1605(00)00366-4

Indira, M., Venkateswarulu, T.C., Abraham Peele, K., Nazneen Bobby, M., Krupanidhi, S., 2019. Bioactive molecules of probiotic bacteria and their mechanism of action: a review. 3 Biotech 9, 306. https://doi.org/10.1007/s13205-019-1841-2

Jain, A., Li, X.H., Chen, W.N., 2018. Similarities and differences in gut microbiome composition correlate with dietary patterns of Indian and Chinese adults. AMB Expr 8, 104. https://doi.org/10.1186/s13568-018-0632-1

Joossens, M., Huys, G., Cnockaert, M., De Preter, V., Verbeke, K., Rutgeerts, P., Vandamme, P., Vermeire, S., 2011. Dysbiosis of the faecal microbiota in patients with Crohn's disease and their unaffected relatives. Gut 60, 631-637. https://doi.org/10.1136/gut.2010.223263 
Kedia, S., Ahuja, V., 2018. Is the emergence of inflammatory bowel disease a prime example of "the third epidemiological transition"? Indian J Gastroenterol 37, 183-185. https://doi.org/10.1007/s12664-018-0852-y

Kedia, S., Ahuja, V., 2017. Epidemiology of Inflammatory Bowel Disease in India: The Great Shift East. Inflamm Intest Dis 2, 102-115. https://doi.org/10.1159/000465522

Kho, Z.Y., Lal, S.K., 2018. The Human Gut Microbiome - A Potential Controller of Wellness and Disease. Front Microbiol 9, 1835. https://doi.org/10.3389/fmicb.2018.01835

Kleppang, A.L., Hagquist, C., 2016. The psychometric properties of the Hopkins Symptom Checklist-10: a Rasch analysis based on adolescent data from Norway. FAMPRJ 33, 740-745. https://doi.org/10.1093/fampra/cmw091

Korterink, J.J., Ockeloen, L., Benninga, M.A., Tabbers, M.M., Hilbink, M., Deckers-Kocken, J.M., 2014. Probiotics for childhood functional gastrointestinal disorders: a systematic review and meta-analysis. Acta Paediatr 103, 365-372. https://doi.org/10.1111/apa.12513

Lakshmi, S.G., Jayanthi, N., Saravanan, M., Ratna, M.S., 2017. Safety assesment of Bacillus clausii UBBC07, a spore forming probiotic. Toxicol Rep 4, 62-71. https://doi.org/10.1016/j.toxrep.2016.12.004

Lee, N.-K., Kim, W.-S., Paik, H.-D., 2019. Bacillus strains as human probiotics: characterization, safety, microbiome, and probiotic carrier. Food Sci Biotechnol 28, 1297-1305. https://doi.org/10.1007/s10068-019-00691-9

Liu, H., Dasgupta, S., Fu, Y., Bailey, B., Roy, C., Lightcap, E., Faustin, B., 2019. Subsets of mononuclear phagocytes are enriched in the inflamed colons of patients with IBD. BMC Immunol 20, 42. https://doi.org/10.1186/s12865-019-0322-z

Loesche, W.J., 1969. Oxygen sensitivity of various anaerobic bacteria. Appl Microbiol 18, 723-727. https://doi.org/10.1128/am.18.5.723-727.1969

Lozupone, C.A., Stombaugh, J.I., Gordon, J.I., Jansson, J.K., Knight, R., 2012. Diversity, stability and resilience of the human gut microbiota. Nature 489, 220-230. https://doi.org/10.1038/nature11550

Mavropoulou, E., Mechie, N.-C., Knoop, R., Petzold, G., Ellenrieder, V., Kunsch, S., Pilavakis, Y., Amanzada, A., 2020. Association of serum interleukin-6 and soluble interleukin-2receptor levels with disease activity status in patients with inflammatory bowel disease: A prospective observational study. PLoS One 15, e0233811. https://doi.org/10.1371/journal.pone.0233811

Neelamraju, J., ratna sudha, M., 2015. Bacillus clausii - The Probiotic of Choice in the Treatment of Diarrhoea. Journal of Yoga \& Physical Therapy 05. https://doi.org/10.4172/2157-7595.1000211

ratna sudha, M., Bhonagiri, S., 2012. Efficacy of Bacillus coagulans strain unique IS-2 in the treatment of patients with acute diarrhea. International Journal of Probiotics and Prebiotics 7, 33-37.

Rocchetti, M.T., Russo, P., Capozzi, V., Drider, D., Spano, G., Fiocco, D., 2021. Bioprospecting Antimicrobials from Lactiplantibacillus plantarum: Key Factors Underlying Its Probiotic Action. International Journal of Molecular Sciences 22, 12076. https://doi.org/10.3390/ijms222112076

Rolfe, R.D., Hentges, D.J., Campbell, B.J., Barrett, J.T., 1978. Factors Related to the Oxygen Tolerance of Anaerobic Bacteria. Appl Environ Microbiol 36, 306-313.

Sanchez-Munoz, F., Dominguez-Lopez, A., Yamamoto-Furusho, J.-K., 2008. Role of cytokines in inflammatory bowel disease. World J Gastroenterol 14, 4280-4288. https://doi.org/10.3748/wjg.14.4280

Sandborn, W.J., Feagan, B.G., Hanauer, S.B., Lochs, H., Löfberg, R., Modigliani, R., Present, D.H., Rutgeerts, P., Schölmerich, J., Stange, E.F., Sutherland, L.R., 2002. A review of 
activity indices and efficacy endpoints for clinical trials of medical therapy in adults with Crohn's disease. Gastroenterology 122, 512-530. https://doi.org/10.1053/gast.2002.31072

Sarlos, P., Kovesdi, E., Magyari, L., Banfai, Z., Szabo, A., Javorhazy, A., Melegh, B., 2014. Genetic update on inflammatory factors in ulcerative colitis: Review of the current literature. World Journal of Gastrointestinal Pathophysiology 5, 304-321. https://doi.org/10.4291/wjgp.v5.i3.304

Schlee, M., Harder, J., Köten, B., Stange, E.F., Wehkamp, J., Fellermann, K., 2008. Probiotic lactobacilli and VSL\#3 induce enterocyte beta-defensin 2. Clin Exp Immunol 151, 528535. https://doi.org/10.1111/j.1365-2249.2007.03587.x

Sokol, H., Pigneur, B., Watterlot, L., Lakhdari, O., Bermúdez-Humarán, L.G., Gratadoux, J.J., Blugeon, S., Bridonneau, C., Furet, J.-P., Corthier, G., Grangette, C., Vasquez, N., Pochart, P., Trugnan, G., Thomas, G., Blottière, H.M., Doré, J., Marteau, P., Seksik, P., Langella, P., 2008. Faecalibacterium prausnitzii is an anti-inflammatory commensal bacterium identified by gut microbiota analysis of Crohn disease patients. Proc Natl Acad Sci U S A 105, 16731-16736. https://doi.org/10.1073/pnas.0804812105

Sudha, M.R., Bhonagiri, S., Kumar, M.A., 2013. Efficacy of Bacillus clausii strain UBBC-07 in the treatment of patients suffering from acute diarrhoea. Benef Microbes 4, 211-216. https://doi.org/10.3920/BM2012.0034

Sudha, M.R., Jayanthi, N., Pandey, D.C., Verma, A.K., 2019. Bacillus clausii UBBC-07 reduces severity of diarrhoea in children under 5 years of age: a double blind placebo controlled study. Benef Microbes 10, 149-154. https://doi.org/10.3920/BM2018.0094

Tursi, A., Brandimarte, G., Papa, A., Giglio, A., Elisei, W., Giorgetti, G.M., Forti, G., Morini, S., Hassan, C., Pistoia, M.A., Modeo, M.E., Rodino', S., D’Amico, T., Sebkova, L., Sacca', N., Di Giulio, E., Luzza, F., Imeneo, M., Larussa, T., Di Rosa, S., Annese, V., Danese, S., Gasbarrini, A., 2010. Treatment of relapsing mild-to-moderate ulcerative colitis with the probiotic VSL\#3 as adjunctive to a standard pharmaceutical treatment: a double-blind, randomized, placebo-controlled study. Am J Gastroenterol 105, 22182227. https://doi.org/10.1038/ajg.2010.218

Upadrasta, A., Pitta, S., Madempudi, R.S., 2016. Draft Genome Sequence of the SporeForming Probiotic Strain Bacillus coagulans Unique IS-2. Genome Announc 4, e00225-16. https://doi.org/10.1128/genomeA.00225-16

Urdaci, M.C., Bressollier, P., Pinchuk, I., 2004. Bacillus clausii probiotic strains: antimicrobial and immunomodulatory activities. $\mathrm{J}$ Clin Gastroenterol 38, S86-90. https://doi.org/10.1097/01.mcg.0000128925.06662.69

Vijay, A., Valdes, A.M., 2021. Role of the gut microbiome in chronic diseases: a narrative review. Eur J Clin Nutr. https://doi.org/10.1038/s41430-021-00991-6

Walmsley, R.S., Ayres, R.C., Pounder, R.E., Allan, R.N., 1998. A simple clinical colitis activity index. Gut 43, 29-32. https://doi.org/10.1136/gut.43.1.29

Wang, W., Chen, L., Zhou, R., Wang, X., Song, L., Huang, S., Wang, G., Xia, B., 2014. Increased proportions of Bifidobacterium and the Lactobacillus group and loss of butyrate-producing bacteria in inflammatory bowel disease. J Clin Microbiol 52, 398406. https://doi.org/10.1128/JCM.01500-13

Weber, K.T., Alipui, D.O., Sison, C.P., Bloom, O., Quraishi, S., Overby, M.C., Levine, M., Chahine, N.O., 2016. Serum levels of the proinflammatory cytokine interleukin-6 vary based on diagnoses in individuals with lumbar intervertebral disc diseases. Arthritis Res Ther 18, 3. https://doi.org/10.1186/s13075-015-0887-8

Zhang, Y.-J., Li, S., Gan, R.-Y., Zhou, T., Xu, D.-P., Li, H.-B., 2015. Impacts of Gut Bacteria on Human Health and Diseases. Int J Mol Sci 16, 7493-7519. https://doi.org/10.3390/ijms16047493 
Zommiti, M., Feuilloley, M.G.J., Connil, N., 2020. Update of Probiotics in Human World: A Nonstop Source of Benefactions till the End of Time. Microorganisms 8, 1907. https://doi.org/10.3390/microorganisms8121907 